\title{
Facilitation of uniform maturation of human retinal pigment epithelial cells through collective movement in culture
}

\author{
Rie Sonoi, Mee-Hae Kim, and Masahiro Kino-oka* \\ Department of Biotechnology, Graduate School of Engineering, Osaka University, 2-1 Yamadaoka,
}

Suita, Osaka 565-0871, Japan

*Corresponding author

Tel: +81-(0)6-6879-7444; fax: +81-(0)6-6879-4246

E-mail address: kino-oka@ bio.eng.osaka-u.ac.jp (M. Kino-oka)

RUNNING TITLE: UNIFORM MATURATION OF RETINAL CELLS

Foornote: Much of this work forms the basis of the Ph.D. dissertation of Rie Sonoi.

(C) 2015. This manuscript version is made available under the Elsevier user license http://www.elsevier.com/open-access/userlicense/1.0/ 


\section{ABSTRCT}

Understanding of the fundamental mechanisms that govern tight junction formation of retinal pigment epithelial (RPE) cells provides surface design strategies for promoting their maturation in culture. RPE cells were cultured to investigate their migratory behavior and the expression of tight junction protein ZO-1 in the central and peripheral regions of a culture vessel. Regardless of locational differences in the culture vessel, the cells at day 1 were elongated in shape, did not form tight junctions, and migrated actively. As the culture progressed, the cells in the central region slowly moved with morphological change of a cobblestone-like shape via interaction between contact cells and exhibiting the shift from random migration to collective movement toward the center, accompanied by tight junction formation. On the other hand, the cells in the peripheral region maintained the random migration at day 5 , meaning spatial heterogeneity in maturation in the vessel. At day 5, RPE cells were incubated in medium with Rac1 inhibitor and the exposure to the Rac1 inhibitor triggered the rapid conversion of migratory behavior from random migration to collective movement toward the center of the vessel, resulting in uniform maturation. These findings indicate that the change in migratory patterns is an important cues and the collective movement toward the center causes the facilitation of uniform maturation in the vessel.

[KEYWORDS: Human retinal pigment epithelial cells, Maturation, Collective movement, Local heterogeneity, Confluence] 


\section{INTRODUCTION}

2 Retinal pigment epithelial (RPE) cells form a part of the blood-retinal barrier and play a pivotal role

3 in maintaining photoreceptor functions and local cellular homeostasis (1). Failure of these functions

4 causes degeneration of the retina and loss of visual function (2). For the recovery of visual function,

5 recent research has been dealing with clinical applications related to the transplantation of RPE cells

6 derived from embryonic stem cells (ES) and induced pluripotent stem (iPS) cells (3-7). The

7 preparation of RPE cells as graft material, entails two stages, namely, expansion and maturation

8 processes. Through the maturation process in confluent culture, RPE cells acquire the typical

9 phenotype through a morphological change from elongated to cobblestone-like shape, resulting in

10 tight junction formation by expressing $\mathrm{ZO}-1$ protein $(8,9)$. Further maturation leads to expression

11 of RPE65 and CRALBP accompanied by $\mathrm{Na}^{+}, \mathrm{K}^{+}$-ATPase pump, and to expression of MITF with

12 pigmentation (10-12).

13 Vegler et al. (12) reported that confluent human RPE cells derived from ES cells had the

14 difference in expression patterns of the markers for RPE cells such as RPE65 and MITF from the

15 center to periphery in the monolayer. In confluent culture, the migratory behavior of epithelial cells

16 is altered in response to cell-cell adhesion level $(13,14)$, suggesting that the initiation for RPE

17 maturation is influenced by their migratory behavior in a culture vessel. The assembly and

18 disassembly of the actin cytoskeleton are further regulated by RhoA, Rac1, and Cdc42 of the Rho

19 small GTPase family (15). Rac1 activation is required to promote actin polymerization and initial

20 cell-cell contact $(16,17)$, while RhoA and Cdc42 are essential for maintaining cell-cell adhesions.

21 In addition, RhoA activity is an antagonistic toward Rac1 activity (18-20). In a previous study, the 
1 confluent cultures were conducted to understand the profiles of RPE cells in the initial state of

2 maturation process and the migration affected spatial heterogeneity of tight junction formation in

3 the vessel (21). In the present study, the spatio-temporal analysis for migratory behavior was further

4 conducted to clarify the initiation of maturation of the cells in the vessel, and the uniform

5 maturation was attempted.

\section{MATERIALS AND METHODS}

7 Culture conditions of human RPE cells Viable human RPE cells (Lot no. 0F3292; Lonza,

8 Walkersville, MD, USA) were seeded at $5.0 \times 10^{4}$ cells $/ \mathrm{cm}^{2}$ on laminin-coated 48 -well plates

9 (culture area in each vessel: $0.95 \mathrm{~cm}^{2}$; diameter: $5.5 \mathrm{~mm}$; Corning Constar, Cambridge, MA, USA)

10 according to a method described previously (21). The laminin-coated surface was prepared by

11 applying a solution of laminin-1 (Sigma-Aldrich, St. Louis, MO, USA) on the bottom surface of

12 48-well plates at $2 \mu \mathrm{g} / \mathrm{cm}^{2}$. The cells were incubated for 5 days in RPE growth medium (One

13 Retinal Pigment Epithelial Cell Medium BulletKit ${ }^{\mathrm{TM}}$; Lonza) at $37{ }^{\circ} \mathrm{C}$ in a humidified $5 \% \mathrm{CO}_{2}$

14 incubator, and the medium was changed every 2 days. The subsequent cells were exposed to Rac1

15 inhibitor NSC23766 (50 $\mu \mathrm{g} / \mathrm{ml}$; Calbiochem, Merck, Darmstadt, Germany) for $12 \mathrm{~h}$. Culture time, $t$,

16 was counted from the addition of the Rac1 inhibitor onwards.

17 Quantitative analyses of migratory behavior and maturation The schematic outline in Fig. 1 Fig. 1

18 shows the procedure of data analysis for the migration rate and direction. Time-lapse images were

19 captured to investigate cell behavior using an image analyzer with a 10x objective lens (IN Cell

20 Analyzer 2000; GE Healthcare, Buckinghamshire, UK). The images were obtained from triplicate

21 samples in 48-well plates, and original images $(1.5 \mathrm{~mm} \times 1.5 \mathrm{~mm})$ at 16 bits in gray scale and 1.83 
1 pixels $/ \mu \mathrm{m}^{2}$ resolution were captured every $20 \mathrm{~min}$ for $12 \mathrm{~h}$ on the entire surface. Multiposition

2 capturing provided tiling images for all cells over the entire surface. For measurement of migration

3 rate at the region of interest (ROI; $300 \mu \mathrm{m} \times 300 \mu \mathrm{m}$ ), the center of the round culture vessel was set

4 at the central position by using the tiling images, and the edge of the vessel was defined as the

5 position to be $5.5 \mathrm{~mm}$ from the center position. Data were obtained from the four ROIs placed at

6 distance from the central position of the culture vessel, $r=0,1.5,3.0$, and $4.5 \mathrm{~mm}$ (Fig. 1). The

7 positional centroids $\left(\mathrm{x}_{i}, \mathrm{y}_{i}\right)$ of each cell in the ROI were determined by using the image processing

8 software (LabVIEW software; National Instruments, Austin, TX, USA) as described previously (21).

9 The migration rate, $V$, of individual cells was determined from the displacement of positional

10 centroids for $12 \mathrm{~h}$. As shown in Fig. 1B, the angle between the vectors from the positional centroid

11 of the cell to the center position of the vessel at 0 and $12 \mathrm{~h}$ after a certain measurement time, $\theta$, was

12 estimated $(0 \leq \theta \leq \pi)$. The average migration rate toward the center, $\bar{V}_{\mathrm{r}}$, was defined as the mean of

$13 V_{\mathrm{r}}=V \cos \theta$, which corresponds to the migration rate toward the center of the vessel. Data were

14 obtained from $300-800$ cells in triplicate cultures.

15 To assess tight junction formation, ZO-1 and cell nuclei were immunostained according to a

16 previously described method (21). In brief, cells from the culture were washed, with PBS were

17 fixed with $4 \%$ paraformaldehyde for $15 \mathrm{~min}$ at $4{ }^{\circ} \mathrm{C}$, and permeabilized by incubation for $5 \mathrm{~min}$ in

$180.2 \%$ Triton X-100. The cells were blocked by treatment with Block Ace (Dainippon Sumitomo

19 Pharma, Osaka, Japan) for $1 \mathrm{~h}$, and treated overnight with anti-rabbit ZO-1 antibody (Abcam,

20 Cambridge, MA, UK) at $4^{\circ} \mathrm{C}$. After the cells were washed with Tris-buffered saline (TBS, Dako,

21 Glostrup, Denmark), they were incubated with Alexa Fluor 488-conjugated goat anti-rabbit IgG 
1 (Life Technologies Corporation, Carlsbad, CA, USA) for $1 \mathrm{~h}$. For nuclear staining, after a TBS,

2 wash, the nuclei were stained with 4',6-diamidino-2-phenylindole (DAPI; Life Technologies

3 Corporation) for $20 \mathrm{~min}$ at room temperature. Fluorescence signal intensities for ZO-1 and DAPI

4 were obtained by excitation at the corresponding wavelengths of 488 and $358 \mathrm{~nm}$, respectively. The

5 staining images of ZO-1 and nuclei were captured using the image analyzer with a $10 \times$ objective

6 lens.

7 To ascertain and quantify the association between maturation and migratory behavior, ZO-1

8 immunostaining images were captured after time-lapse observation in the same positions where

9 migration rates were analyzed. The cells were divided into two groups, namely, ZO-1-positive and

10 ZO-1-negative groups, according to the criteria depicted in Fig. 1C. The frequencies of

11 ZO-1-positive and ZO-1-negative cells, $F_{\mathrm{Z}}$ and $F_{\mathrm{N}}$, respectively, were estimated. In addition, the

12 ZO-1-positive and ZO-1-negative cells were assumed to exhibit the collective movement and

13 random migration, respectively. Thus, the frequency of cells with collective movement and random

14 migration is equivalent to be $F_{\mathrm{Z}}$ and $F_{\mathrm{N}}$, respectively. Furthermore, the frequency of cells with

15 collective movement toward the center, $F_{Z c}$, was determined to be the frequency of ZO-1-positive

16 cells migrating toward the center of the vessel $\left(V_{\mathrm{r}}>0\right)$.

17 Statistical analysis All data were obtained from triplicate cultures, and were expressed as means

18 with standard deviations. Comparisons between the groups were determined by one-way analysis of

19 variance and the Tukey-Kramer post-hoc test. $p$ values $<0.05$ were considered statistically

20 significant. 
2 Maturation of RPE cells in culture Spatio-temporal analysis was carried out in the central and

3 peripheral regions at $r=0$ and $4.5 \mathrm{~mm}$, respectively. Immunostaining images of ZO- 1 and cell

4 nuclei were captured at the same positions. After seeding, the cells in both regions exhibited

5 elongated shapes and migrated actively (Movie S1). As culture time elapsed, the cells in the central

6 region shrank, and their random migration decreased. At day 5, the cells in the central region made

7 a cobblestone-like shape, and they moved collectively with maintaining cell-cell contact. In

8 contrast, the cells in the peripheral region at day 5 maintained the elongated shape and random

9 migration, and the morphological change from elongated to cobblestone-like shape in the peripheral

10 region was delayed. In both regions, positive ZO-1 expression was found in cells exhibiting

11 collective movement. Thus, it is considered that the migratory behaviors of ZO-1-positive and

12 ZO-1-negative cells were distinguished to be collective movement and random migration,

13 respectively.

14 Effect of migratory inhibition on maturation The timing of inhibition for migration was

15 determined based on the data obtained from temporal analysis (Fig. 2). As the culture proceeded, it

16 was found that the absolute values, $\left|V_{\mathrm{r}}\right|$, decreased in the central region. At day 3 , the

17 ZO-1-positive cells with collective movement increased in the central region, although

18 ZO-1-negative cells with active random migration are major in the peripheral region. At day 5,

19 ZO-1 positive cells with collective movement were major $\left(F_{\mathrm{Z}}=0.75\right)$ and the distribution is in the

20 range of $V_{\mathrm{r}}>0$ in the central region, meaning that cells made collective movement toward the

21 center in the vessel. On the other hand, cells in the peripheral region showed lower $F_{\mathrm{Z}}$ level and 
1 higher $\left|V_{\mathrm{r}}\right|$ level, meaning the exhibition of collective movement and active random migration. To

2 make detail clarification, the effect of migration behaviors on the initiation of maturation, the Rac1

3 inhibitor was added at day $5(t=0)$, and their migration rates and the directions in the central and

4 peripheral regions from $t=0$ to $t=12 \mathrm{~h}$ were examined.

As shown in Fig. 3, cells migrated slowly through interaction between neighbor cells, which

6 had ZO-1 expression, under both conditions (Fig. 3a and b). In the culture without the Rac1

7 inhibitor, the migration rates of the cells in the peripheral region were higher than those in the

8 central region, and cells migrating randomly and actively had negative ZO-1 expression (Fig. 3c),

9 showing spatial heterogeneity for initiation of maturation. In the culture with the Rac1 inhibitor, the

10 random migration of the cells in the peripheral region decreased and became unidirectional

11 movement toward the center of the vessel, and their ZO-1 expression was positive, similar to the

12 central region (Fig. 3d).

13 The frequencies of cells with collective movement and migration rate toward the center were

14 assessed along with radius direction (Fig. 4). At $r=0$ in culture without the Rac1 inhibitor, the

15 average migration rate was $\bar{V}_{\mathrm{r}}=0.0 \pm 1.7 \mu \mathrm{m} / \mathrm{h}$ (Fig. $4 \mathrm{a}$ ). The standard deviations at $r=0-4.5 \mathrm{~mm}$

16 in culture without the Rac1 inhibitor increased with increasing the distance from the center of the

17 vessel (Fig. 4a-d). At $r=4.5 \mathrm{~mm}, \mathrm{ZO}-1$-negative cells increased and the broad distribution of $V_{\mathrm{r}}$

18 were observed ranging from $V_{\mathrm{r}}=-8.0 \mu \mathrm{m} / \mathrm{h}$ to $V_{\mathrm{r}}=8.0 \mu \mathrm{m} / \mathrm{h}$ (Fig. $4 \mathrm{~d}$ ), causing random migration.

19 This means that spatial heterogeneity of migratory behavior exists in the vessel. In the culture with

20 the Rac1 inhibitor (Fig. 4e-h), the distributions in $V_{\mathrm{r}}$ at $r=0-4.5 \mathrm{~mm}$ were significantly more

21 narrow than those in culture without the Rac1 inhibitor (Fig. 4a-d). Especially, the standard 
1 deviation of $V_{\mathrm{r}}$ at $r=4.5 \mathrm{~mm}$ in the culture with the Rac1 inhibitor (Fig. 4h) was one fifth lower

2 than that of the culture without the inhibitor (Fig. 4d). In addition, under both conditions, the

3 frequency in cells with collective movement and $V_{\mathrm{r}}>0$ is more than half, meaning that the

4 migration toward the center were caused by collective movement.

5 For understanding of the effect of collective movement toward the center on the maturation, the

6 frequency of cells with collective movement toward the center, $F_{\mathrm{Z}}$ and $F_{\mathrm{Zc}}$ at $r=0,1.5,3.0$, and 4.5

$7 \mathrm{~mm}$ in the vessel at $t=12 \mathrm{~h}$ were compared. As shown in Fig. $5, F_{Z}$ and $F_{Z \mathrm{c}}$ at the central region in

8 culture without Rac1 inhibitor was the highest $\left(F_{\mathrm{Z}}=0.75 \pm 0.10\right.$ and $\left.F_{\mathrm{Zc}}=0.71 \pm 0.14\right)$,

9 respectively, and these values decreased toward the periphery. Moreover, the difference between

10 values of $F_{\mathrm{Z}}$ and $F_{\mathrm{Zc}}$ increased along with radius direction, being $F_{\mathrm{Z}}=0.47 \pm 0.10$ and $F_{\mathrm{Zc}}=0.21 \pm$

110.10 at $r=4.5 \mathrm{~mm}$. In culture with Rac1 inhibitor, $F_{Z \mathrm{c}}$ at $r=4.5 \mathrm{~mm}$ was 3.5 -fold higher than that

12 of culture without Rac1 inhibitor, and $F_{Z c}$ reached high level of $0.72-0.78$ in whole vessel, meaning

13 uniform maturation in the vessel. These results revealed that the migratory inhibition enhanced the

14 tight junction formation with rapid conversion of random migration to collective migration,

15 facilitating the movement toward the center as well as maturation.

\section{DISCUSSION}

Migratory behavior is known to change in response to cellular phenotype, and the migratory

behavior of individual RPE cells has been shown to be closely related to tight junction formation associated with their maturation (21). In the present study, time-lapse observation and 
1 cultured on laminin-coated surfaces were found to be elongated and exhibited random migration in

2 the confluent state (Movie S1). As shown in Fig. 6, this random migration is considered to occur Fig. 6

3 through the formation of lamellipodia at the leading edge of the cells. RPE cells cultured on

4 laminin-coated surfaces secrete fibronectin, and integrin $\alpha 5 \beta 1$ binding to fibronectin is the critical

5 step involved in cell migration and growth (22). Danen et al. (23) reported that the binding of

6 integrin $\beta 1$ to fibronectin increased Rac1 activity by autophosphorylation of focal adhesion kinase

7 (FAK), causing random migration by the formation of lamellipodia. These reports indicate that the

8 binding of integrin to fibronectin induces Rac1 activation by the autophosphorylation of FAK in

9 immature cells, which explains the random migration observed in the present study. During the

10 development of the adhesions between cells, their expression of ZO-1 increased, and their random

11 migration decreased. RPE cells generally express N-cadherin as their dominant cadherin $(24,25)$,

12 and p120 catenin binding to the cadherin localized to the cell membrane is known to cause

13 stabilization of cell-cell adhesions by suppressing endocytosis $(26,27)$. Tyrosine phosphorylation

14 of p120 catenin can induce the unbinding of cadherin complexes on the cell membrane (28), and

15 then the cytosolic p120 catenin is complexed with RhoA-GDP, thereby inhibiting RhoA activation

16 (29). Furthermore, RPE cells transfected with p120 catenin siRNA increase RhoA activity (30),

17 suggesting that the localization of p120 catenin to cadherin led to the increase in RhoA activity in

18 the present study. RhoA activation is known to decrease Rac1 activity (31) and induce myosin

19 activation by phosphorylating the light chain of myosin II, which leads to the assembly of tight

20 junction proteins (32). Considering these findings, it is most likely that RhoA activation caused the

21 assembly of tight junctions in our study. As maturation progresses, the actomyosin contraction 
1 network and ZO-1 proteins assemble at the apical sides $(33,34)$, and RhoA activation increases

2 actomyosin contraction $(19,35)$. These cells are found to move collectively while exhibiting the

3 cobblestone-like shape (Movie S1). The cobblestone-like cells are supposed to increase dark

4 pigmentation, because microphthalmia-associated transcription factor induces the expression of the

5 tyrosinase gene controlling pigment synthesis $(11,36)$. Considering these findings, it can be stated

6 that the mature cells had migratory behavior, with the maintenance of cell-cell adhesions, which is

7 named as 'collective movement' $(37,38)$. Simultaneous actomyosin contraction in whole vessel is

8 considered to cause collective movement toward the center. These suggested that the transition from

9 random migration to collective movement is an important event to initiate the maturation.

10 Location-dependent analysis from the center toward the periphery of the vessel revealed that

11 the extent of the initiation for maturation gradually decreased along this path, and that the

12 frequencies of the cells having random migration increased at the same time (Fig. 4 and 5),

13 suggesting that the random migration in the vessel is responsible for locational heterogeneity of the

14 maturation in culture. In the present study, the Rac1 inhibitor was applied to suppress the random

15 migration of the cells in the vessel, and the cells in whole vessel slowly moved toward the center of

16 the vessel, although the inhibition of Rac1 actively had no effect on the initiation of maturation

17 against the cells already exhibiting collective movement (Fig. 5). The frequency of the maturation

18 in culture with Rac1 inhibitor, $F_{Z c}$, increased, similarly to that at the center. This uniform

19 maturation is considered to occur owing to the change in migratory behavior from random

20 migration to collective movement toward the center in the peripheral region. As illustrated in Fig. 6,

21 the inhibition of lamellipodium formation through suppression of Rac1 activity was curbed the 
1 random migration, leading to the stabilization of cell-cell adhesions. Subsequently, RhoA activation

2 appears to have induced the assembly of tight junction proteins, and the cells in the periphery

3 reached a greater frequency of the maturation similarly to the cells at the center of the vessel. Taken

4 together, our findings reveal that suppression of random migration can facilitate switching to

5 collective movement toward the center, causing uniform maturation in the vessel.

In conclusion, RPE cells were cultured to characterize the location-dependent profiles of the

7 migratory behavior governing their maturation in culture. Time-lapse observation and ZO-1

8 immunostaining at the same positions where migration was analyzed were performed to examine

9 the association between migration and maturation. ZO-1-negative cells exhibited elongated shape

10 with random migration, while ZO-1-positive cells exhibited cobblestone-like shape with collective

11 movement. The migratory behavior of the random migration and collective movement implied

12 locational heterogeneity of maturation in the vessel. The suppression of migration resulted in the

13 facilitation of uniform maturation by collective movement toward the center. The present study

14 suggests that conversion of migration into collective movement toward the center is critical for

15 regulating the maturation of the cells in the vessel.

\section{ACKNOWLEDGMENTS}

17 This work was supported by the Strategic Promotion of Innovative Research and Development

18 Program of the Japan Science and Technology Agency, and the JSPS Japanese-German Graduate

19 Externship. This work was also supported by the Strategic Promotion of Innovative Research and

20 Development (S-Innovation) Program of the Japan Science and Technology Agency (JST). 
2 1. Strauss, O.: The retinal pigment epithelium in visual function, Physiol. Rev., 85, 845-881 (2005).

4

2. Melville, H., Carpiniello, M., Hollis, K., Staffaroni, A., and Golestaneh, N.: Stem cells: a new paradigm for disease modeling and developing therapies for age-related macular degeneration, J. Transl. Med., 11, 53 (2013).

3. Schwartz, S.D., Hubschman, J.P., Heilwell, G., Cardenas, V.F., Pan, C.K., Ostrick, R.M., Mickunas, E., Gay, R., Klimanskaya, I., and Lanza, R.: Embryonic stem cell trials for macular degeneration: a preliminary report, Lancet., 379, 713-720 (2012).

4. Li, Y., Tsai, Y.T., Hsu, C.W., Erol, D., Yang, J., Wu, W.H., Davis, R.J., Egli, D., and Tsang, S.H.: Long-term safety and efficacy of human-induced pluripotent stem cell (iPS) grafts in a preclinical model of retinitis pigmentosa, Mol. Med., 18, 1312-1319 (2012).

5. Kanemura, H., Go, M.J., Nishishita, N., Sakai, N., Kamao, H., Sato, Y., Takahashi, M., and Kawamata, S.: Pigment epithelium-derived factor secreted from retinal pigment epithelium facilitates apoptotic cell death of iPSC, Sci. Rep., 3, 2334 (2013).

6. Kamao, H., Mandai, M., Okamoto, S., Sakai, N., Suga, A., Sugita, S., Kiryu, J., and Takahashi, M.: Characterization of human induced pluripotent stem cell-derived retinal pigment epithelium cell sheets aiming for clinical application, Stem Cell Reports., 2, 205-218 (2014).

7. Borooah, S., Phillips, M.J., Bilican, B., Wright, A.F., Wilmut, I., Chandran, S., Gamm, D., and Dhillon, B.: Using human induced pluripotent stem cells to treat retinal disease, prog. 
Retin. Eye. Res., 37, 163-181 (2013).

8. McKay, B.S., Irving, P.E., Skumatz, C.M., and Burke, J.M.: Cell-cell adhesion molecules and the development of an epithelial phenotype in cultured human retinal pigment epithelial cells, Exp. Eye. Res., 65, 661-671 (1997).

9. Maminishkis, A., Chen, S., Jalickee, S., Banzon, T., Shi, G., Wang, F.E., Ehalt, T., Hammer, J.A., and Miller, S.S.: Confluent monolayers of cultured human fetal retinal pigment epithelium exhibit morphology and physiology of native tissue, Invest. Ophthalmol. Vis. Sci., 47, 3612-3624 (2006).

10. Bok, D.: The retinal pigment epithelium: a versatile partner in vision, J. Cell. Sci. Suppl., 17, 189-195 (1993).

11. Burke, J.M.: Epithelial phenotype and the RPE: is the answer blowing in the Wnt?, Prog. Retin. Eye. Res., 27, 579-595 (2008).

12. Vugler, A., Carr, A.J., Lawrence, J., Chen, L.L., Burrell, K., Wright, A., Lundh, P., Semo, M., Ahmado, A., Giasm, C., da Cruz, L., Moore, H., Andrews, P., Walsh, J., and Coffey, P.: Elucidating the phenomenon of HESC-derived RPE: anatomy of cell genesis, expansion and retinal transplantation, Exp. Neurol., 214, 347-361 (2008).

13. Doxzen, K., Vedula, S.R., Leong, M.C., Hirata, H., Gov, N.S., Kabla, A.J., Ladoux, B., and Lim, C.T.: Guidance of collective cell migration by substrate geometry, Integr. Biol., 5, 1026-1035 (2013).

14. Ng, M.R., Besser, A., Danuser, G., and Brugge, J.S.: Substrate stiffness regulates cadherin-dependent collective migration through myosin-II contractility, J. Cell. Biol., 199, 
545-563 (2012).

15. Jaffe, A.B., and Hall, A.: Rho GTPases: biochemistry and biology, Annu. Rev. Cell. Dev. Biol., 21, 247-269 (2005).

16. Nobes, C.D., and Hall, A.: Rho GTPases control polarity, protrusion, and adhesion during cell movement, J. Cell Biol., 144, 1235-1244 (1999).

17. Yamada, S., and Nelson, W.J.: Localized zones of Rho and Rac activities drive initiation and expansion of epithelial cell-cell adhesion, J. Cell. Biol., 178, 517-527 (2007).

18. Itoh, M., Tsukita, S., Yamazaki, Y., and Sugimoto, H.: Rho GTP exchange factor ARHGEF11 regulates the integrity of epithelial junctions by connecting ZO-1 and RhoA-myosin II signaling, Proc. Natl. Acad. Sci. USA., 109, 9905-9910 (2012).

19. Omelchenko, T., and Hall, A.: Myosin-IXA regulates collective epithelial cell migration by targeting RhoGAP activity to cell-cell junctions, Curr. Biol., 22, 278-288 (2012).

20. McCormack, J., Welsh, N.J., and Braga, V.M.M.: Cycling around cell-cell adhesion with Rho GTPase regulators, J. Cell Sci., 126, 379-391 (2013).

21. Sonoi, R., Kim, M.H., and Kino-oka, M.: Locational heterogeneity of maturation by changes in migratory behaviors of human retinal pigment epithelial cells in culture, J. Biosci. Bioeng., 119, 107-112 (2015).

22. Li, R., Maminishkis, A., Zahn, G., Vossmeyer, D., and Miller, S.S.: Integrin alpha5beta1 mediates attachment, migration, and proliferation in human retinal pigment epithelium: relevance for proliferative retinal disease, Invest. Ophthalmol. Vis. Sci., 50, 5988-5996 (2009).

23. Danen, E.H., van Rheenen, J., Franken, W., Huveneers, S., Sonneveld, P., Jalink, K., and 
Sonnenberg, A.: Integrins control motile strategy through a Rho-cofilin pathway, J. Cell. Biol., 169, 515-526 (2005).

24. Burke, J.M., Cao, F., Irving, P.E., and Skumatz, C.M.: Expression of E-cadherin by human retinal pigment epithelium: delayed expression in vitro, Invest. Ophthalmol. Vis. Sci., 40, 2963-2970 (1999).

25. Kaida, M., Cao, F., Skumatz, C.M., Irving, P.E., and Burke, J.M.: Time at confluence for human RPE cells: effects on the adherens junction and in vitro wound closure, Invest. Ophthalmol. Vis. Sci., 41, 3215-3224 (2000).

26. Ishiyama, N., Lee, S.H., Liu, S., Li, G.Y., Smith, M.J., Reichardt, L.F., and Ikura, M.: Dynamic and static interactions between p120 catenin and E-cadherin regulate the stability of cell-cell adhesion, Cell., 141, 117-128 (2010).

27. Nanes, B.A., Chiasson-MacKenzie, C., Lowery, A.M., Ishiyama, N., Faundez, V., Ikura, M., Vincent, P.A., and Kowalczyk, A.P.: p120-catenin binding masks an endocytic signal conserved in classical cadherins, J. Cell. Biol., 199, 365-380 (2012).

28. Ozawa, M., and Ohkubo, T.: Tyrosine phosphorylation of p120(ctn) in v-Src transfected L cells depends on its association with E-cadherin and reduces adhesion activity, J Cell. Sci., 114, 503-512 (2000).

29. Anastasiadis, P.Z., Moon, S.Y., Thoreson, M.A., Mariner, D.J., Crawford, H.C., Zheng, Y., and Reynolds, A.B.: Inhibition of RhoA by p120 catenin, Nat. Cell. Biol., 2, 637-644 (2000).

30. Chen, H.C., Zhu, Y.T., Chen, S.Y., and Tseng, S.C.: Selective activation of p120ctn-Kaiso signaling to unlock contact inhibition of ARPE-19 cells without epithelial-mesenchymal 
transition, PLoS. One., 7, e36864 (2012).

31. Parri, M., and Chiarugi, P.: Rac and Rho GTPases in cancer cell motility control, Cell. Commun. Signal., 8, 23-37 (2010).

32. Terry, S.J., Zihni, C., Elbediwy, A., Vitiello, E., Leefa Chong San, I.V., Balda, M.S., and Matter, K.: Spatially restricted activation of RhoA signalling at epithelial junctions by p114RhoGEF drives junction formation and morphogenesis, Nat. Cell. Biol., 13, 159-166 (2011).

33. Miyoshi, J., and Takai, Y.: Structural and functional associations of apical junctions with cytoskeleton, Biochim. Biophys. Acta., 1778, 670-691 (2008).

34. Geisen, P., McColm, J.R., King, B.M., and Hartnett, M.E.: Characterization of barrier properties and inducible VEGF expression of several types of retinal pigment epithelium in medium-term culture, Curr. Eye. Res., 31, 739-748 (2006).

35. Levayer, R., and Lecuit, T.: Biomechanical regulation of contractility: spatial control and dynamics, Trends Cell Biol., 22, 61-81 (2012).

36. Tachibana, M.: MITF: a stream flowing for pigment cells, Pigment. Cell. Res., 13, 230-240 (2000).

37. Menko, A.S., Bleaken, B.M., and Walker, J.L.: Regional-specific alterations in cell-cell junctions, cytoskeletal networks and myosin-mediated mechanical cues coordinate collectivity of movement of epithelial cells in response to injury, Exp. Cell. Res., 322, 133-148 (2014).

38. Murrell, M., Kamm, R., and Matsudaira, P.: Substrate viscosity enhances correlation in epithelial sheet movement, Biophys. J., 101, 297-306 (2011). 


\section{Figure legends}

2 FIG. 1 Procedure of data analyses for the directional migration rate of the cells at each region of interest (ROI) in a culture vessel.

FIG. 2 Frequencies of cells with collective movement and random migration against the migration rate toward the center of individual cells in the vessel at day 1 ( $a$ and d), 3 (b and e), and 5 (c and f). Closed and open bars show frequencies of cells with collective movement and random migration, respectively. The migration rate toward the center, $V_{\mathrm{r}}$, of individual cells was determined from the displacement of positional centroids for $12 \mathrm{~h}$. The average migration rate, $\bar{V}_{\text {r }}$, with standard deviation was calculated from the data obtained from 300-800 cells in triplicate cultures.

FIG. 3 ZO-1-negatitve and ZO-1-positive cells with their migration rate and direction in culture without (a and c) or with the Rac1 inhibitor (b and d) at $t=12 \mathrm{~h}(300 \mu \mathrm{m} \times 300 \mu \mathrm{m}$ fields of view). The direction having $0 \leq \theta<\pi / 2$ and $\pi / 2 \leq \theta \leq \pi$ were considered migratory behavior toward the center (yellow vector) and periphery (red vector), respectively, of the vessel. The green and blue circles show ZO-1-negative cells with random migration and ZO-1-positive cells with collective movement, respectively. The green or blue circles without arrows depict cells with a migration rate of $V=0.0$. The scale bars show $50 \mu \mathrm{m}$.

FIG. 4 Frequencies of cells with collective movement and random migration $\left(F_{\mathrm{Z}}\right.$ and $F_{\mathrm{N}}$, respectively) against migration rate toward the center at $r=0(\mathrm{a}, \mathrm{e}), 1.5(\mathrm{~b}, \mathrm{f}), 3.0(\mathrm{c}, \mathrm{g})$, and $4.5 \mathrm{~mm}(\mathrm{~d}, \mathrm{~h})$ in culture without Rac1 inhibitor $(\mathrm{a}-\mathrm{d})$ and with the Rac1 inhibitor $(\mathrm{e}-\mathrm{h})$. Closed and open bars show frequencies of cells with collective movement and random 
migration, respectively. The average migration rate and standard deviation were calculated from the data obtained from $700-800$ cells in triplicate cultures at day 5 .

FIG. 5 Frequencies of cells with collective movement and with collective movement toward the center $\left(F_{\mathrm{Z}}\right.$ and $F_{\mathrm{Zc}}$, respectively) at day 5 . Open and closed circles show $F_{\mathrm{Z}}$ values in culture without or with the Rac1 inhibitor, respectively. $F_{\mathrm{Z}}$ and $F_{\mathrm{Zc}}$ in each region were obtained from three independent experiments $(n=3)$. Statistical significance among the groups was determined by one-way analysis of variance (ANOVA) and the Tukey-Kramer post-hoc test $(* p<0.05, * * p<0.01)$.

FIG. 6 Schematic diagram of possible mechanism of the maturation based on migratory behavior of RPE cells in culture. 


\section{Procedure of data analyses}

\section{A. Multiposition capturing of ZO-1 and bright-field images}

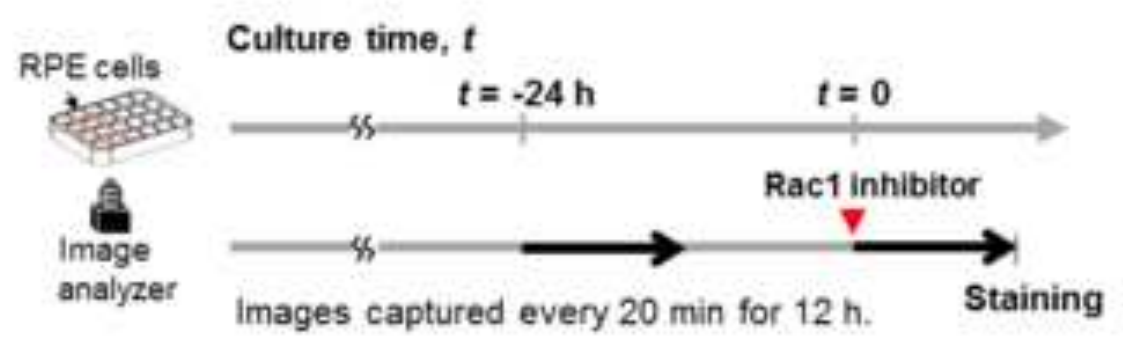

\section{Judgment of ZO-1-positive and ZO-1-negative cells}

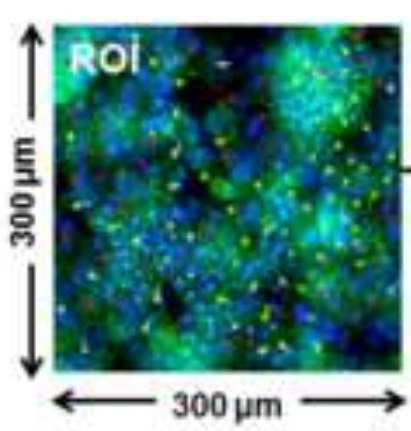

Immunostaining image for $\mathrm{ZO}-1$ and nuclei in same region where migration rate was analyzed.

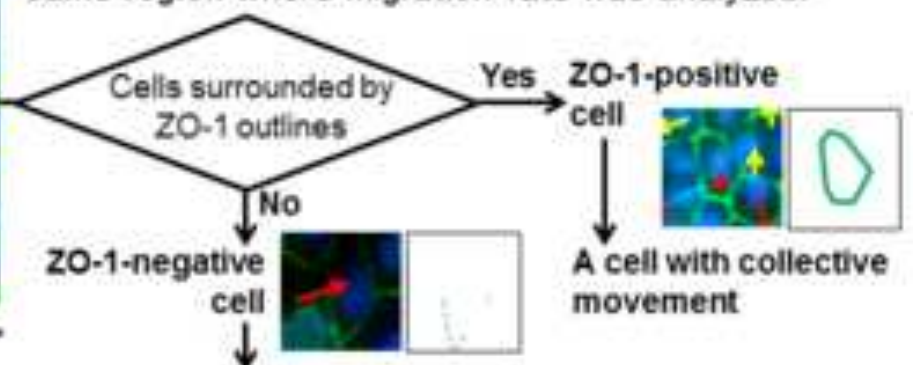

B. Assessment of cells migrating toward the central or peripheral position

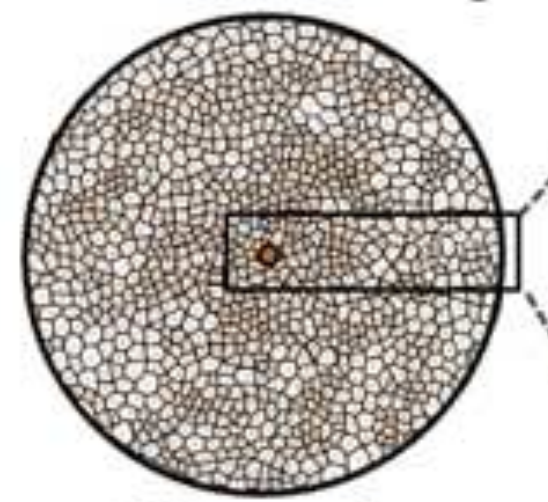

Culture vessel
Central position

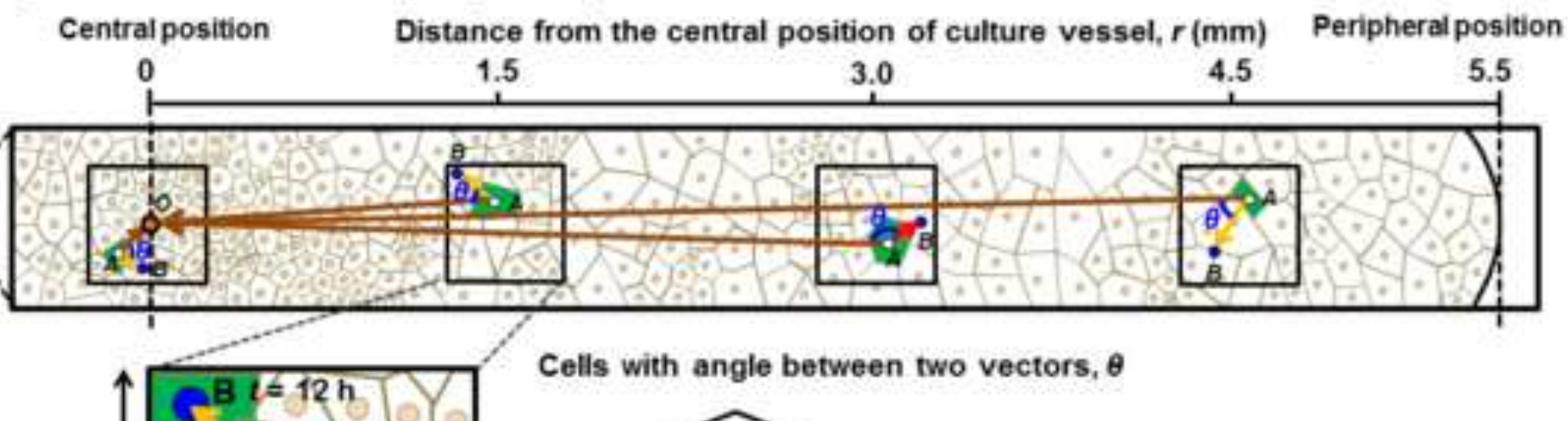

Distance from the central position of culture vessel, $r(\mathrm{~mm})$

Peripheral position
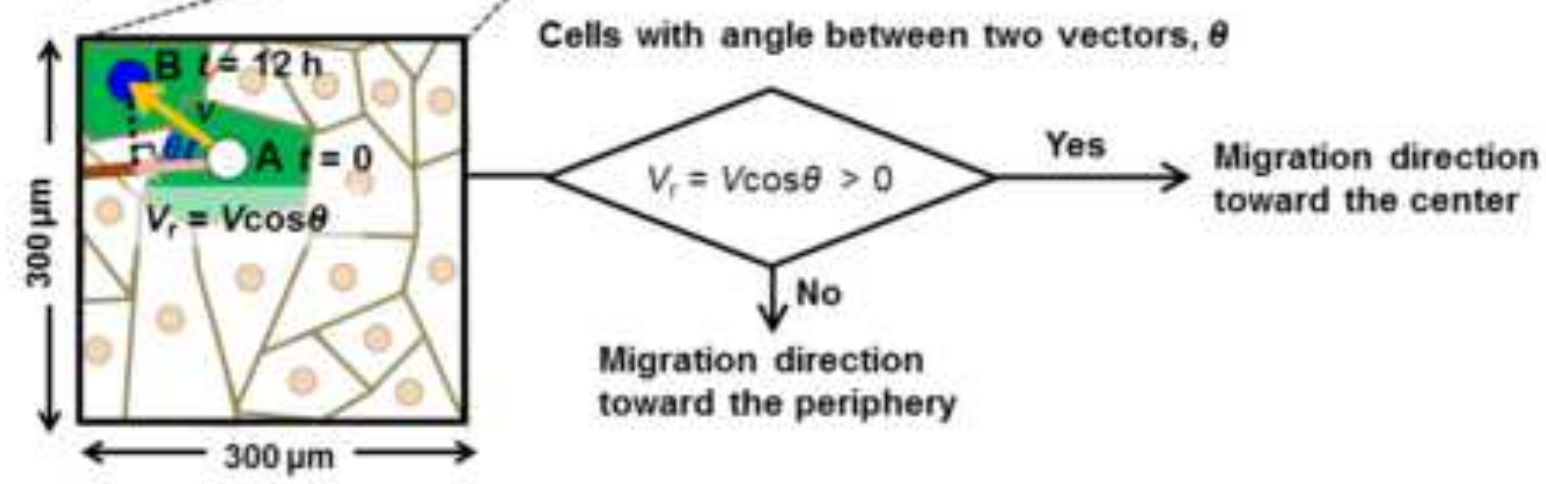

Migration direction

toward the periphery 


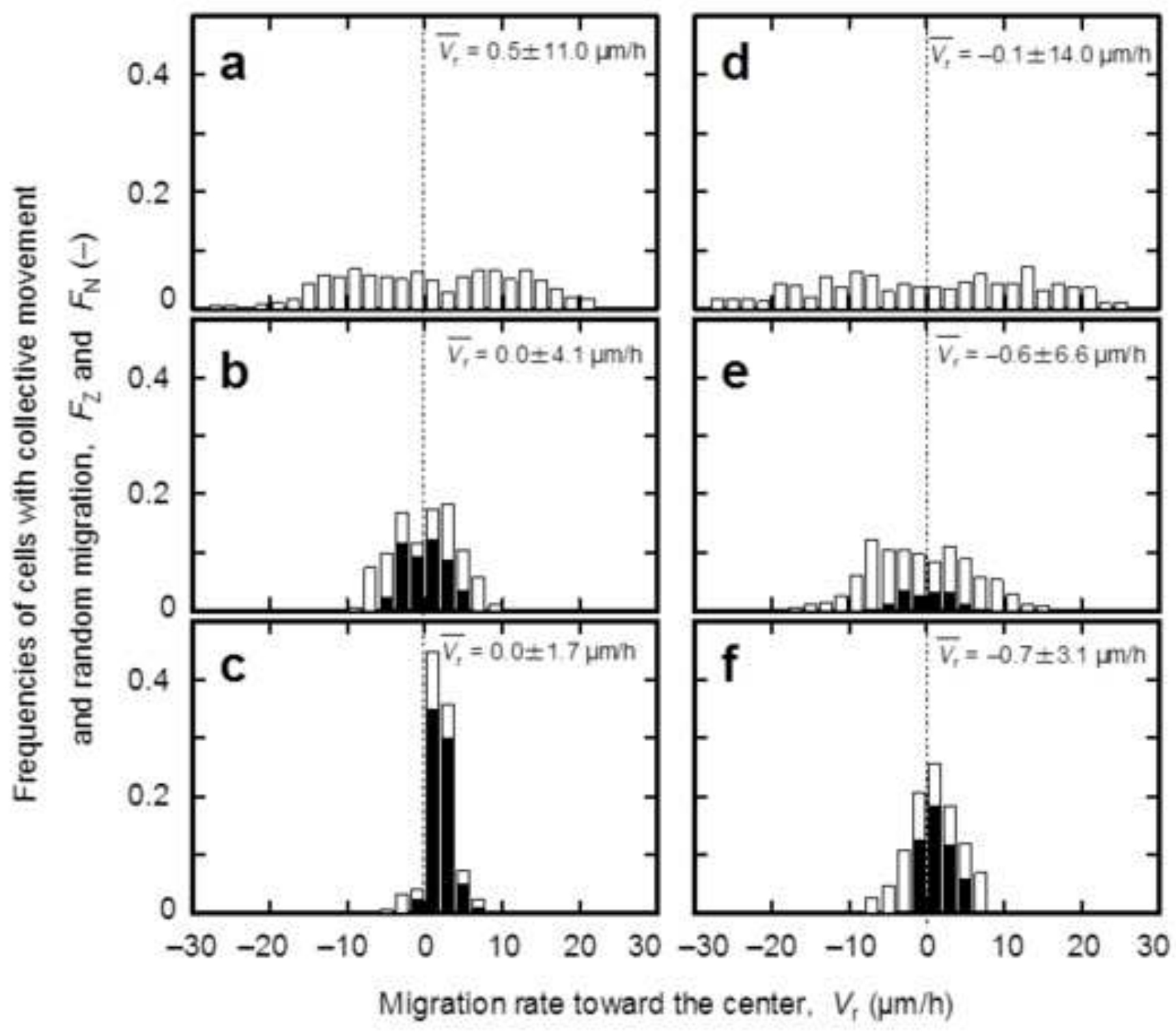



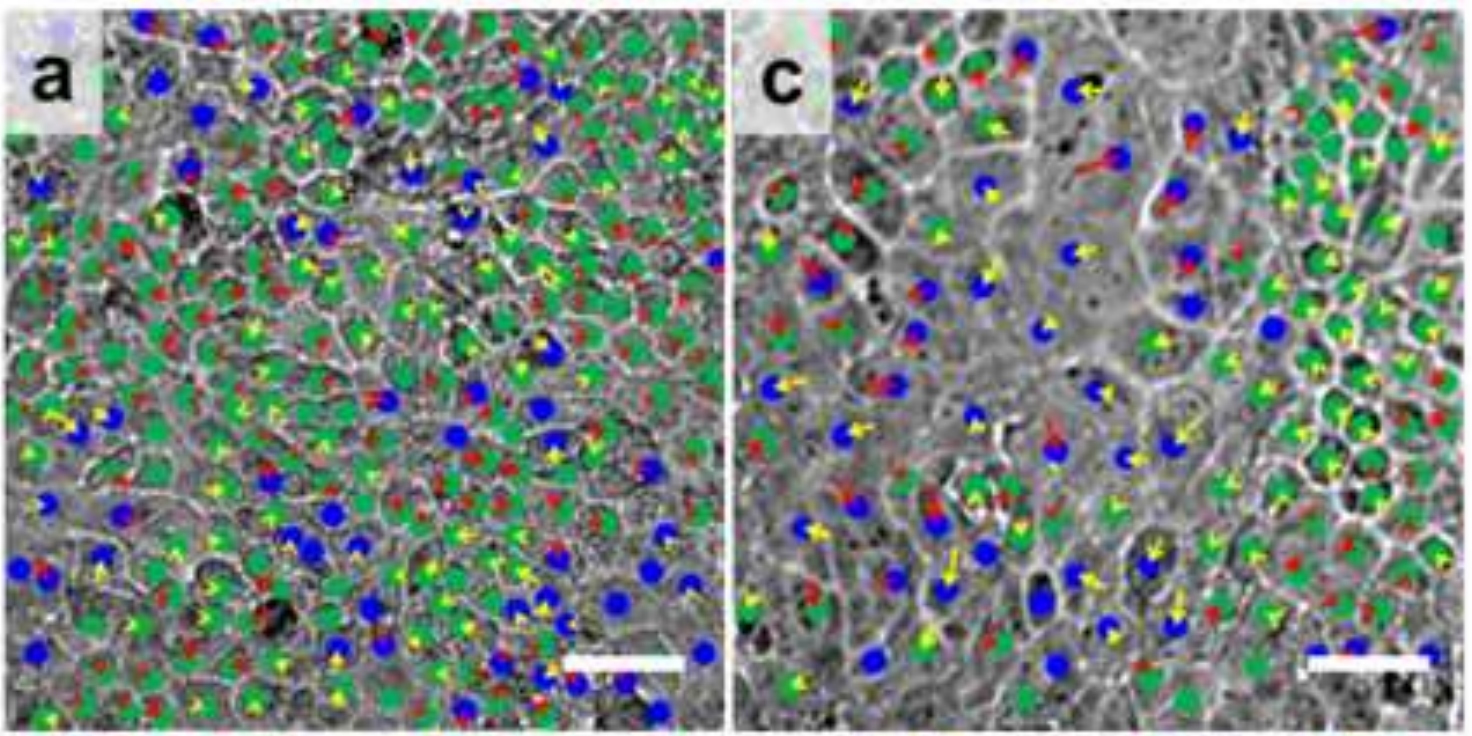

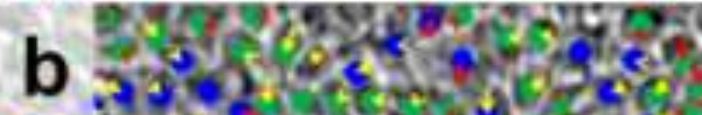

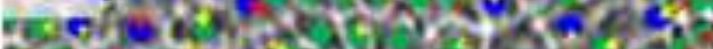

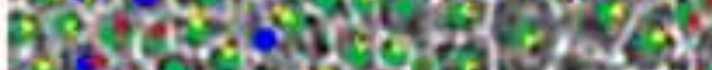

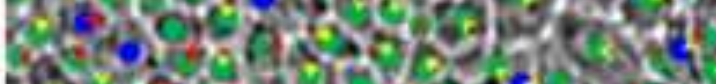

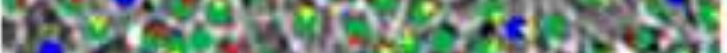
20, 2 is

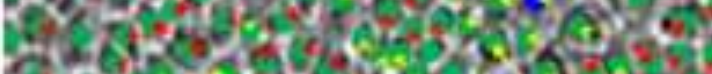

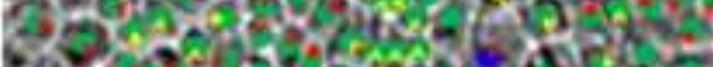

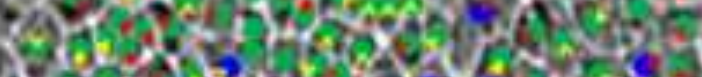

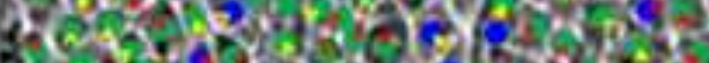

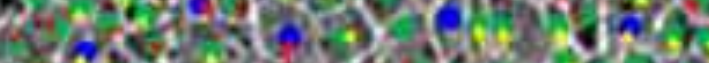
Xis.t.

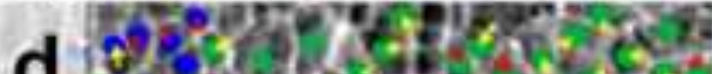

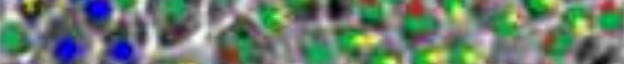

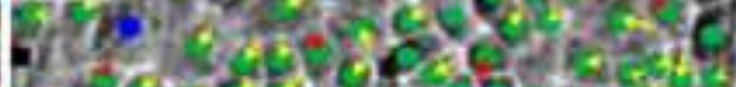

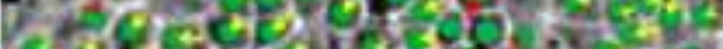

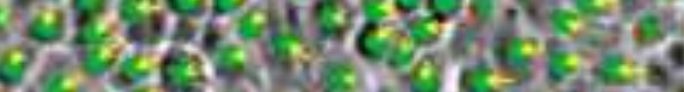

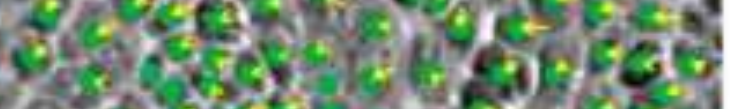

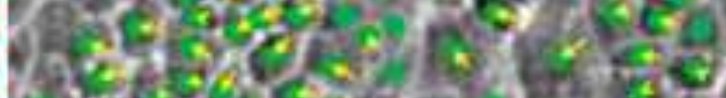

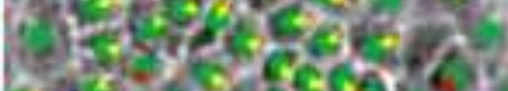

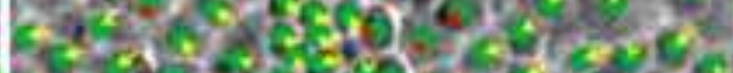

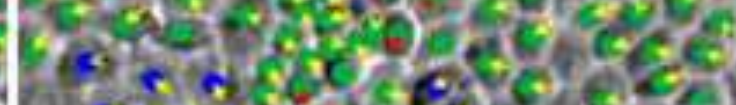

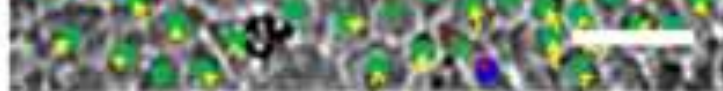

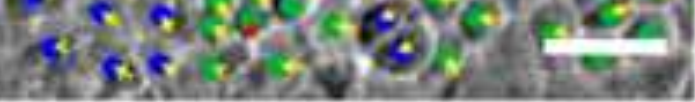




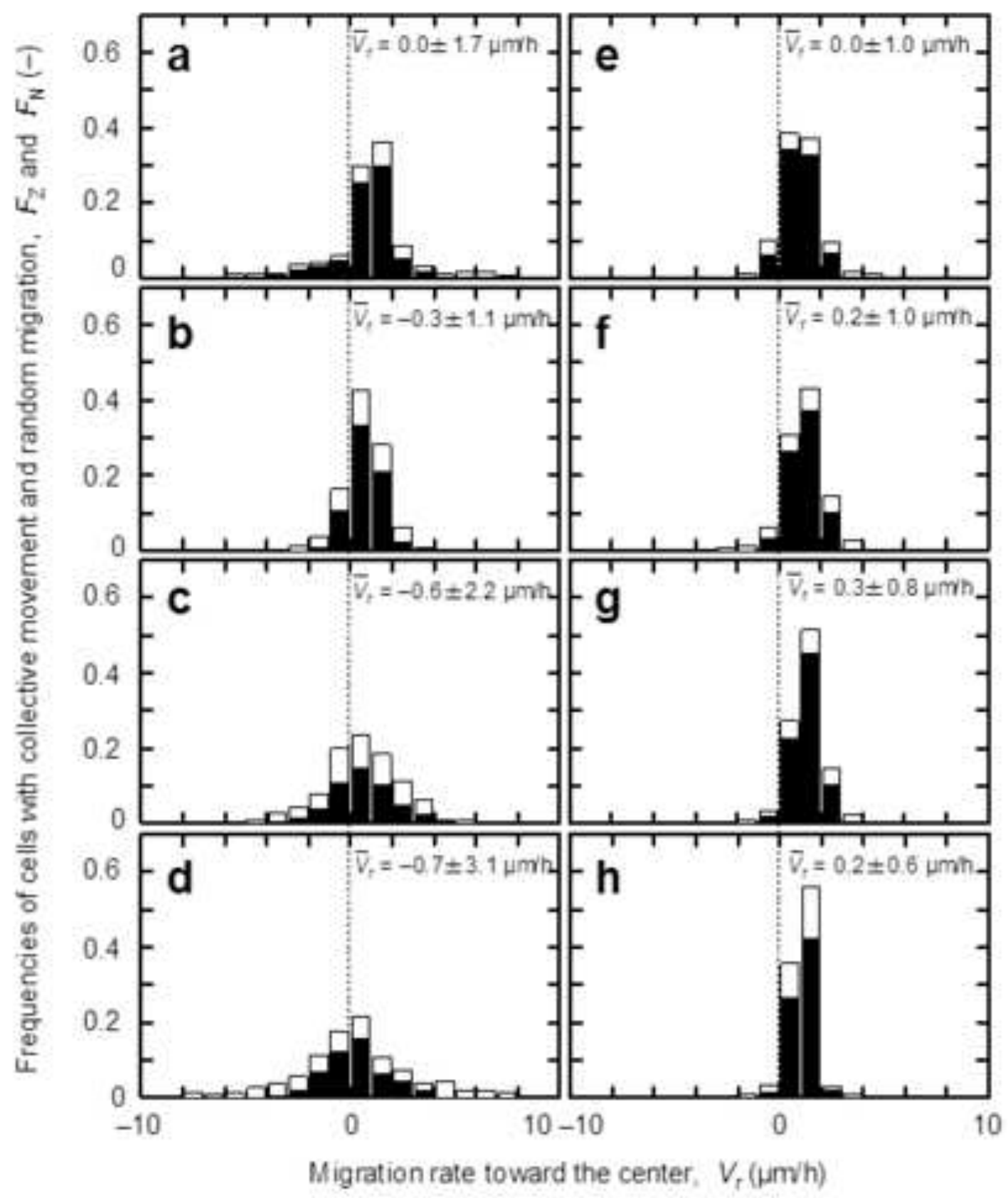




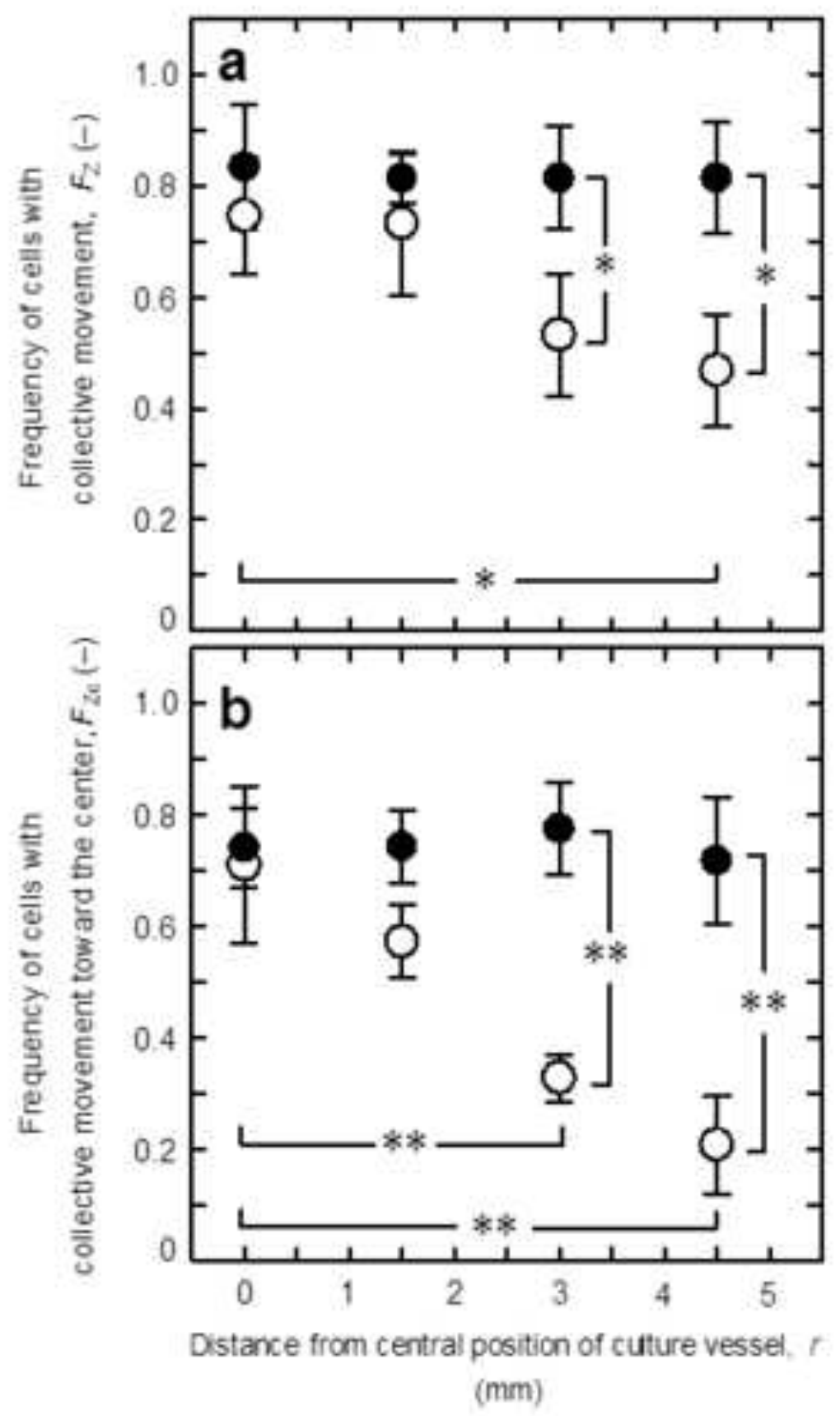




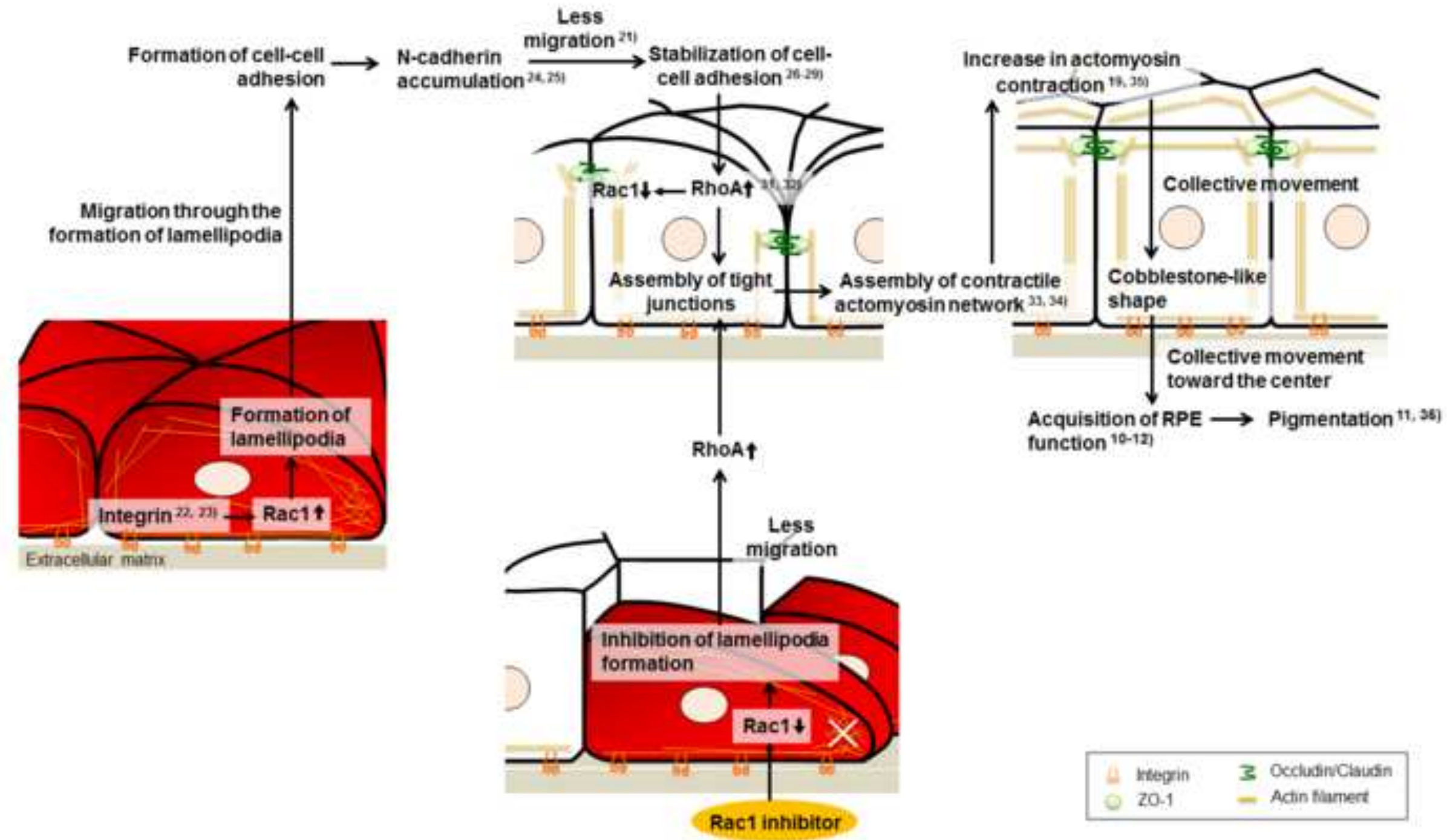

